\title{
Implementation of a Drift-Flux Model in SAM for Modeling of Passively Transported Gas in Molten Salt Reactors
}

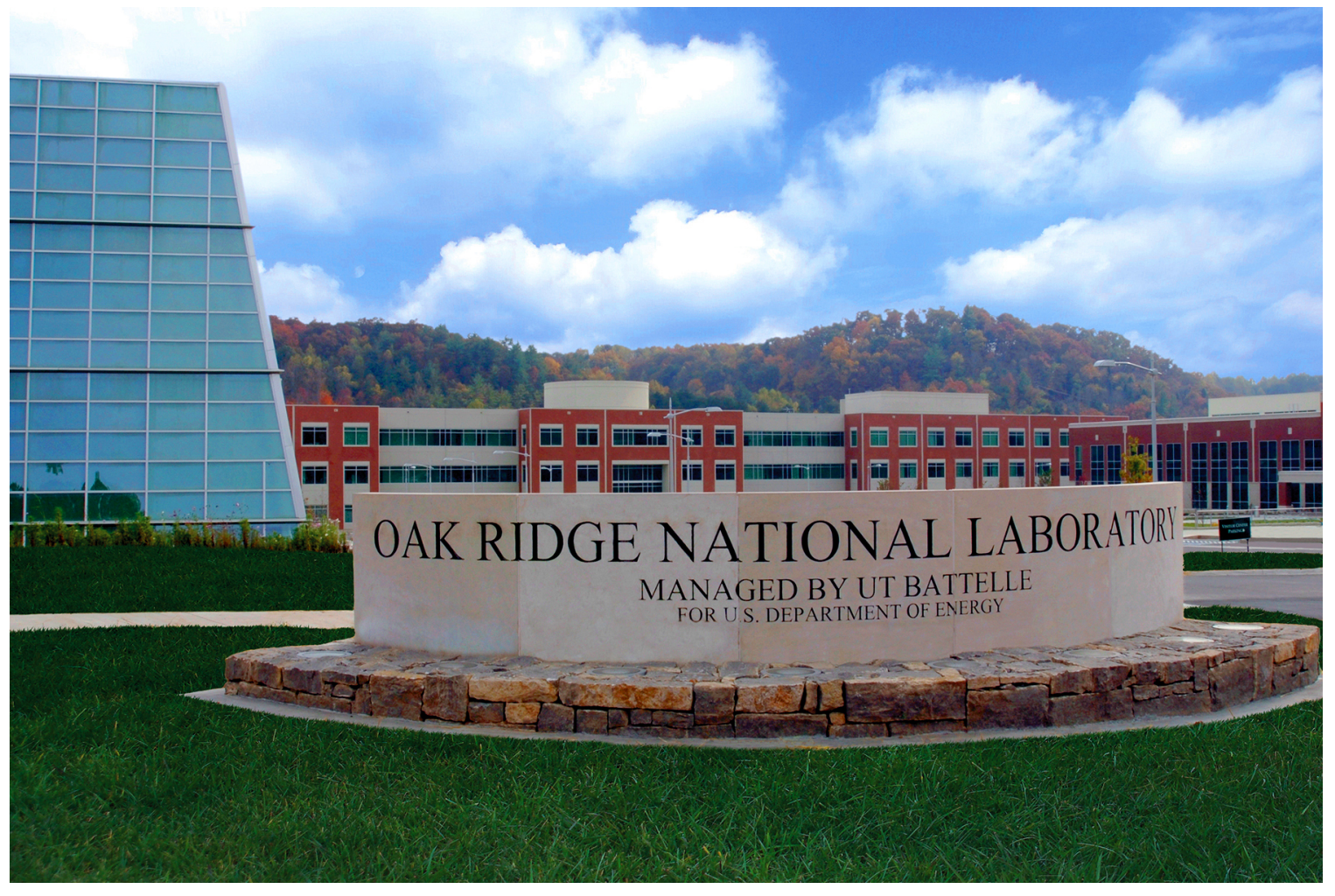

\section{R. Salko* \\ T. Mui \\ R. $\mathrm{Hu}^{\dagger}$ \\ E. Merzari ${ }^{+}$}

September, 2021

* Oak Ridge National Laboratory, Oak Ridge, TN

† Argonne National Laboratory, Chicago, IL

+ The Pennsylvania State University, University Park, PA
Approved for public release.

Distribution is unlimited.

\section{OAK RIDGE




\section{DOCUMENT AVAILABILITY}

Reports produced after January 1, 1996, are generally available free via US Department of Energy (DOE) SciTech Connect.

Website: www.osti.gov/

Reports produced before January 1, 1996, may be purchased by members of the public from the following source:

National Technical Information Service

5285 Port Royal Road

Springfield, VA 22161

Telephone: 703-605-6000 (1-800-553-6847)

TDD: $703-487-4639$

Fax: 703-605-6900

E-mail: info@ntis.gov

Website: http://classic.ntis.gov/

Reports are available to DOE employees, DOE contractors, Energy Technology Data Exchange representatives, and International Nuclear Information System representatives from the following source:

Office of Scientific and Technical Information

PO Box 62

Oak Ridge, TN 37831

Telephone: 865-576-8401

Fax: 865-576-5728

E-mail: report@osti.gov

Website: http://www.osti.gov/contact.html

This report was prepared as an account of work sponsored by an agency of the United States Government. Neither the United States Government nor any agency thereof, nor any of their employees, makes any warranty, express or implied, or assumes any legal liability or responsibility for the accuracy, completeness, or usefulness of any information, apparatus, product, or process disclosed, or represents that its use would not infringe privately owned rights. Reference herein to any specific commercial product, process, or service by trade name, trademark, manufacturer, or otherwise, does not necessarily constitute or imply its endorsement, recommendation, or favoring by the United States Government or any agency thereof. The views and opinions of authors expressed herein do not necessarily state or reflect those of the United States Government or any agency thereof. 
Nuclear Energy and Fuel Cycle Division

\title{
Implementation of a Drift-Flux Model in SAM for Modeling of Passively Transported Gas
} in Molten Salt Reactors

\author{
Author(s) \\ R. Salko* T. Mui ${ }^{\dagger}$ R. Hu ${ }^{\dagger}$ E. Merzari ${ }^{+}$
}

September, 2021

\footnotetext{
* Oak Ridge National Laboratory, Oak Ridge, TN

$\dagger$ Argonne National Laboratory, Chicago, IL

+ The Pennsylvania State University, University Park, PA
}

Prepared by

OAK RIDGE NATIONAL LABORATORY

Oak Ridge, TN 37831-6283

managed by

UT-Battelle LLC

for the

US DEPARTMENT OF ENERGY

under contract DE-AC05-00OR22725 



\section{CONTENTS}

LIST OF FIGURES $\ldots \ldots \ldots \ldots \ldots \ldots \ldots \ldots \ldots \ldots \ldots \ldots$

LIST OF TABLES $\ldots \ldots \ldots \ldots \ldots \ldots \ldots \ldots \ldots \ldots \ldots \ldots \ldots \ldots$ vii

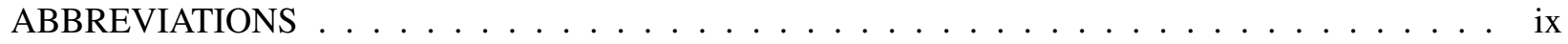

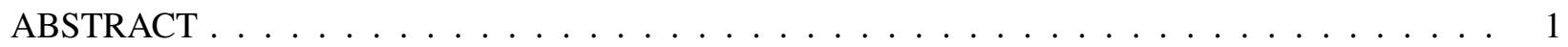

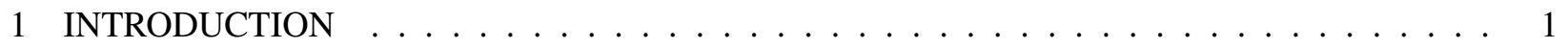

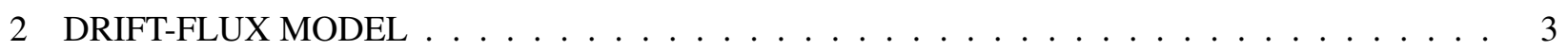

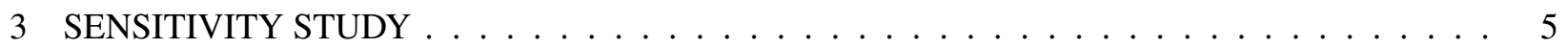

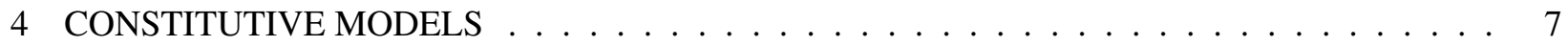

5 MODEL IMPLEMENTATION $\ldots \ldots \ldots \ldots \ldots \ldots \ldots$

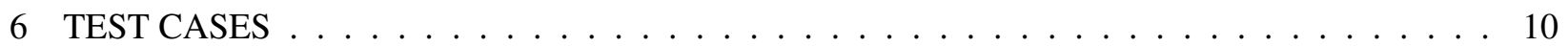

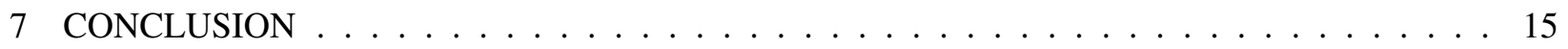

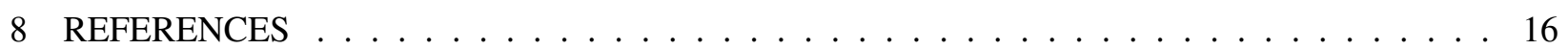





\section{LIST OF FIGURES}

1 Axial species concentration distribution in Case 1 test using the base model and drift-flux model. . . . . . . . . . . . . . . . . . . . . . . 11

2 Axial species mass flow rate distribution in Case 1 test using the base model and drift-flux model. . . . . . . . . . . . . . . . . . . . . . . 11

3 Axial species gas volume fraction distribution in Case 1 test using the base model and drift-flux model. . . . . . . . . . . . . . . . . . . . . 12

4 Axial species gas bubble radius distribution in Case 1 test using the base model and drift-flux model. . . . . . . . . . . . . . . . . . . . . . . 12

5 Axial species gas interfacial area distribution in Case 1 test using the base model and drift-flux model. . . . . . . . . . . . . . . . . . . . . 13

6 Axial gas volume fraction distribution in heated channel test using the base model and drift-flux model. . . . . . . . . . . . . . . . . . . . . . . 14 



\section{LIST OF TABLES}

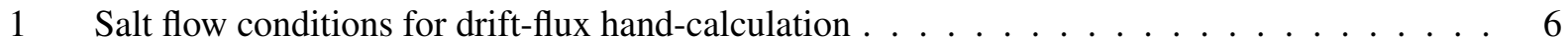

2 Comparison of SAM results and hand-calculated gas phase properties for the unheated single-channel test case . . . . . . . . . . . . . . . . . . . 13 



\section{ABBREVIATIONS}

BWR boiling water reactor

FLiBe fluoride-lithium-beryllium

FLiNaK fluoride-lithium-sodium-potassium

INL Idaho National Laboratory

LWR light-water reactor

MOOSE Multiphysics Object Oriented Simulation Environment

M\&S modeling and simulation

MSR molten salt reactor

MSRE Molten Salt Reactor Experiment

NEAMS Nuclear Energy Advanced Modeling and Simulation

ORNL Oak Ridge National Laboratory

SAM System Analysis Module

T/H thermal hydraulics 



\begin{abstract}
The Nuclear Energy Advanced Modeling and Simulation (NEAMS) program is further developing the Multiphysics Object Oriented Simulation Environment (MOOSE) system thermal hydraulics (T/H) code, System Analysis Module (SAM), to include modeling of molten salt reactor (MSR) designs. MSR designs in which the fuel is dissolved in the salt coolant poses unique modeling and simulation (M\&S) challenges because fission products - some of which will be noncondensable gasses - travel with the coolant, thus allowing for deposition and interaction throughout the entire system flow loop. This behavior must be modeled to successfully understand MSR system behavior during normal and off-normal operation events. In past work, SAM has been extended to include a passive species transport capability for modeling of MSR fission products that travel with the salt coolant. This work extends the model to include a drift-flux model to capture the tendency of the gas phase to move at a different velocity than the bulk liquid.

Additional constitutive models have also been added for calculation of gas volume fraction, bubble radius, and interfacial area, which will be important for calculation of species phase migration in future coupling activities. Testing has been performed to ensure that the new model agrees with expected values for several simple models, but additional future work will be required to test the model for more complex geometries that will be needed for full-scale MSR simulations.
\end{abstract}

\title{
1 INTRODUCTION
}

The goal of this work package is to build on the species transport capability in the Multiphysics Object Oriented Simulation Environment (MOOSE)-based thermal hydraulics (T/H) systems application System Analysis Module (SAM) (Hu et al. [2021]), for applications to molten salt reactor (MSR) designs that have the fuel dissolved into the coolant. This design poses unique chemistry and corrosion challenges that must be understood, including production and removal of fission products, transmutation of isotopes, and production and deposition of noble metals. In the Nuclear Energy Advanced Modeling and Simulation (NEAMS) program, the modeling and simulation (M\&S) tool being developed for this application is the MOOSE application Mole, which models transport of dissolved species in molten salt, including production, deposition, and interaction with other species (Lee et al. [2021]). The SAM application is used for $\mathrm{T} / \mathrm{H} \mathrm{M} \& \mathrm{~S}$ of single-phase flow in advanced reactor coolant systems, which includes MSR designs. For the MSR problem, Mole and SAM will be coupled so that Mole can make use of the $\mathrm{T} / \mathrm{H}$ solution obtained by SAM for performing the species transport solution.

Note that species of interest in an MSR system can include both dissolved and gaseous components. For example, xenon-135 is a fission gas produced during normal operation and must be removed because of its high thermal neutron absorption cross section, which can cause degraded economic performance of the reactor. It was observed during the operation of the Molten Salt Reactor Experiment (MSRE) facility at Oak Ridge National Laboratory (ORNL) in the late 1960s that introducing a dispersed helium gas into the system can improve the removal efficiency of xenon from the system (Engel and Steffy [1971]). A summary of the removal process and the findings in the MSRE can be found in Taylor (Taylor et al. [2022]). Briefly put, helium cover gas that is in contact with the free salt surface in the pump bowl is entrained during normal operation of the reactor, leading to a finely dispersed field of small helium bubbles (low void of less than $1 \%$, typically) that are entrained in the salt. The xenon tends to conglomerate in the helium gas, which is then removed from the system for later filtration. This process is also referred to as "gas sparging." 
This project seeks to improve on the species transport capabilities of SAM by implementing a modeling capability for noncondensable gasses, which will interact with system fission products. Note that SAM has an internal species mass transport solver, which will model passive transport of any number of user-defined species. This capability can be used to model the noncondensable gas, including its production at the injection site in addition to convection and diffusion in the system. The current SAM model does not consider the possibility of the species to travel at a different velocity than the bulk fluid velocity. Despite the volume fraction of the gas being small, it is possible that there may be a discrepancy between the gas velocity and bulk velocity, which can impact the species transport solution. A drift-flux model is presented in Section 2, which can be used for predicting the relative velocity between phases in a two-phase system. A hand calculation was performed using this model (documented in Section 3), which demonstrates that the gas velocity can differ considerably from the bulk fluid velocity. Because of this finding, the drift-flux model was implemented into SAM. In addition to the drift-flux model, it is noted that prediction of void and interfacial area is also needed for Mole to determine the rate of fission product migration into the gas phase. The method for calculation of these terms is presented in Section 4. Model implementation considerations are presented in Section 5. Finally, results from testing of the new models after implementation into SAM are shown in Section 6. 


\section{DRIFT-FLUX MODEL}

The drift-flux model that will be implemented into SAM follows from the model described by Zuber and Findlay Zuber and Findlay [1965]. This model proposes that the dispersed (gas) phase velocity can be represented in relative terms to the bulk flow velocity, as shown below.

$$
v_{g}=j+V_{g j}
$$

Here, $v_{g}$ is the gas velocity, $j$ is the volumetric flux of the mixture, and $V_{g j}$ is the drift velocity of the gas phase with respect to the bulk flow. Considering that Eq. (1) applies to local flow behavior, and in keeping with the nomenclature used by Zuber, it must be averaged over the node solved by SAM. An area-weighted average is used to represent solution variables, which is defined as follows:

$$
\langle F\rangle=\frac{1}{A} \int_{A} F d A
$$

In the equation, $F$ is an arbitrary value that is being area-weight-averaged, and $A$ represents the area. The angle-bracket nomenclature denotes area averaging. In addition to this, a void-weighted averaging will also be used for determining two-phase properties, as defined below.

$$
\bar{F}=\frac{\langle\alpha F\rangle}{\langle\alpha\rangle} .
$$

Applying this averaging to Eq. (1) yields the following.

$$
\overline{v_{g}}=\frac{\langle\alpha j\rangle}{\langle\alpha\rangle}+\frac{\left\langle\alpha V_{g j}\right\rangle}{\langle\alpha\rangle} .
$$

By multiplying the first term in the equation by $\langle j\rangle /\langle j\rangle$, the equation can be expressed in terms of the distribution parameter, $C_{0}$.

$$
\overline{v_{g}}=C_{0}\langle j\rangle+\frac{\left\langle\alpha V_{g j}\right\rangle}{\langle\alpha\rangle}
$$

Here, the definition of the distribution parameter is considered as follows.

$$
C_{0}=\frac{\langle\alpha j\rangle}{\langle\alpha\rangle\langle j\rangle}
$$

Physically, the distribution parameter represents the radial distribution of void; a value of 1 means that void is uniform across the flow area cross-section, and a value that is greater than 1 means that the void is concentrated in the center of the flow channel. The benefit of expressing the vapor velocity in terms of the distribution parameter is that previous authors have developed constitutive relationships for this parameter from experimental data that can be utilized. Ishii (Ishii [1977]) provides an expression for this parameter 
for bubbly flow conditions using experimental data from several authors. This is expressed in terms of the max-to-mean ratio of velocity of a single-phase flow in a tube, $C_{\infty}$.

$$
C_{\infty}=\lim \frac{\langle\alpha j\rangle}{\langle\alpha\rangle\langle j\rangle}=1.393-0.0155 \ln \left(\frac{G D}{\mu_{f}}\right)
$$

This expression holds true when void approaches zero. When the density ratio between gas and liquid approaches unity, the distribution parameter, $C_{0}$, shall approach unity. Considering this, the following expression was proposed.

$$
C_{0}=C_{\infty}-\left(C_{\infty}-1\right) \sqrt{\rho_{g} / \rho_{f}}
$$

Returning to Eq. (5), the second term can be simplified to $V_{g j}$, considering that this calculation will be performed for a single node, where only one void fraction is available. Similar to $C_{0}$, a constitutive model can also be used for determination of $V_{g j}$. To determine an expression for this, Zuber considers a gas phase traveling in an upward flow in an infinite media that is undisturbed by the presence of other particles. In this case, the gas will flow at a terminal velocity, which can be determined as a function of the buoyancy force acting on the bubble. For bubbly flow, Zuber recommends the following expression.

$$
V_{g j}=1.53\left[\frac{\sigma g \Delta \rho}{\rho_{l}^{2}}\right]^{1 / 4} .
$$

It is noted that both the distribution parameter (Eq. [7]) and the drift velocity (Eq. [9]) were qualified using steam-water or air-water mixtures, so work will be needed to qualify them for salt/gas mixtures. Looking back to Eq. (5), the last remaining term is the bulk fluid volumetric flux, $j$. Considering that for MSR applications void will be very low (i.e., less than $1 \%$ ), it is reasonable to assume that $j$ is equal to the liquid velocity predicted by SAM during its regular governing equation solve. 


\section{SENSITIVITY STUDY}

To determine the importance of modeling the gas species velocity independent of the bulk velocity, a hand calculation was performed. An upward-flowing fluoride-lithium-beryllium (FLiBe) mixture was considered with a helium noncondensable gas uniformly distributed in the mixture. Flow conditions and salt properties used for the calculation are shown in Table 1. Note that the salt surface tension was obtained from an expression given for FLiBe, which is presented in an Idaho National Laboratory (INL) report Sohal et al. [2010], and reproduced here as Eq. (10). The inlet temperature of $707.15 \mathrm{~K}$ was used in this equation to obtain the surface tension presented in Table 1, but note that the authors did state that the range of applicability of the correlation is $732.2-4498.8 \mathrm{~K}$.

$$
\sigma=0.26921\left[1-\left(\frac{T}{4498.8}\right)^{1} .64\right]
$$

The MSRE technical report did not specify a helium injection rate, which would be needed to determine the flow quality; however, it did specify a range of void fractions for the experiments. For the ${ }^{235} \mathrm{U}$ runs, void ranged from $2 \cdot 10^{-4}$ to $4.5 \cdot 10^{-4}$ and for the ${ }^{233} \mathrm{U}$ runs, void ranged from $5 \cdot 10^{-3}$ to $6 \cdot 10^{-3}$. Using a void of $6 \cdot 10^{-3}$ as the target, the required quality was iteratively determined from the void/quality/slip relationship (later introduced as Eq. [12]) to be $2.130 \cdot 10^{-9}$. This corresponds to a gas mass flow rate of $2.70 \times 10^{-10} \mathrm{~kg} \mathrm{~s}^{-1}$ considering that the salt mass flow rate for the stated conditions is $0.127 \mathrm{~kg} \mathrm{~s}^{-1}$. Using the drift-flux model presented in the previous section, the gas velocity will be $0.561 \mathrm{~m} \mathrm{~s}^{-1}$, which is a slip ratio of 2.63. This substantial amount of phase slip shows that the gas velocity may have an important impact on the gas mass transport solution and that further consideration is warranted. 
Table 1. Salt flow conditions for drift-flux hand-calculation

\begin{tabular}{lrr}
\hline Parameter & Value & Source \\
\hline Pressure & $101.325 \mathrm{kPa}$ & \\
Temperature & $707.15 \mathrm{~K}$ & 1 \\
Salt velocity & $0.2134 \mathrm{~m} \mathrm{~s}^{-1}$ & 2 \\
Salt density & $2068.1 \mathrm{~kg} \mathrm{~m}^{-3}$ & 3 \\
He density & $2.76 \times 10^{-4} \mathrm{~kg} \mathrm{~m}^{-3}$ & 4 \\
Salt viscosity & $7.78 \times 10^{-3} \mathrm{~kg} \mathrm{~s}^{-1} \mathrm{~m}^{-1}$ & 1 \\
Salt surface tension & $2.6 \times 10^{-1} \mathrm{~N} \mathrm{~m}^{-1}$ & 5 \\
Channel flow area & $2.88 \times 10^{-4} \mathrm{~m}^{2}$ & 6 \\
Hydraulic diameter & $1.59 \times 10^{-2} \mathrm{~m}$ & 6 \\
\hline
\end{tabular}

1. Table 2.1 in Kedl [1972]

2. Section 2.2 in Kedl [1972]

3. SAM FLiBe properties

4. Ideal gas law at stated temperature and pressure

5. Sohal et al. [2010]

6. MSRE flow channel geometry 


\section{CONSTITUTIVE MODELS}

To determine the rate of mass transport between liquid and the gas phase, Mole will need to know the interfacial area of the gas phase, which requires knowledge of the total gas volume fraction and bubble size distribution. The interfacial area can be estimated by assuming that the bubbles will have a spherical shape, which leads to the following expression for surface area per unit volume for a single bubble.

$$
A_{i}^{\prime \prime \prime}=\frac{\alpha 4 \pi r^{2}}{4 / 3 \pi r_{b}^{3}}=\frac{3 \alpha}{r_{b}}
$$

Here, $A_{i}^{\prime \prime \prime}$ is the surface area per unit volume, $\alpha$ is the volume fraction of the gas, and $r_{b}$ is the bubble

radius. To obtain the volume fraction, the relationship between void, quality, and slip, shown in Eq. (12), is utilized.

$$
\alpha=\frac{x}{C_{0}\left(x+\rho_{v} / \rho_{l}(1-x)\right)+\rho_{v} v_{g j} / G} .
$$

Here, $x$ is the flow quality and $G$ is the bulk mass flux. For simplicity, the phase slip was replaced with the drift-flux relationship in this equation. The flow quality will be calculated as the gas mass flow rate divided by the bulk mass flow rate. The bubble radius can be calculated from the Weber number, which is defined as follows.

$$
W e=\frac{\rho v^{2} l}{\sigma} .
$$

Here, the characteristic length, $l$, will be the bubble diameter. The velocity, $v$, will be the relative velocity between the gas and liquid, or $v_{g}-v_{l}$. The critical Weber number for the fluid is the ratio between inertial and surface tension forces, and it therefore can be used to determine the size at which bubbles break up. This number therefore sets a maximum size for bubbles and, in this way, the bubble radius can be calculated as follows.

$$
r_{b}=\frac{W e \sigma}{2 \rho_{l} v_{r}^{2}}
$$

The Weber number will need to be determined for molten salt applications. For light-water reactor (LWR) applications, a value of 10 is commonly used (Salko et al. [2019]). Because the surface tension for water at boiling water reactor (BWR) conditions will be less than the value found for MSR applications ([Eq. 10]), it is expected this value will be lower than 10. One study (Chavez [2020]) indicates that this value was 1.59 and 4.36 for an experimental fluoride-lithium-sodium-potassium (FLiNaK) system with helium bubbles flowing upward. Considering this, a value of 3.0 is utilized in SAM; however, it is noted that this value will need to be revisited in the future. From the form of Eq. (14), it can be seen that the radius will have a linear relationship with the Weber number. An inversely linear relationship exists for the interfacial area.

Note that a lower limit must be placed on the relative velocity, $v_{r}$, to prevent dividing by zero, and, more importantly, an upper limit must be placed on the bubble radius to prevent it from becoming unrealistically 
high. It was assumed in Engel (Engel and Steffy [1971]) that the bubble diameter was between 5 and 20 mils $(0.13-0.5 \mathrm{~mm})$ for the MSRE conditions. Using this as a guide, and considering the values obtained by the drift-flux model (to be calculated in Section 6), an upper limit of $2 \mathrm{~mm}$ was placed on bubble radius. 


\section{MODEL IMPLEMENTATION}

The existing mass transport model in SAM will be used for modeling of the noncondensable gas in the system. However, the model will be modified so the mass transport can use a drift-flux velocity instead of the bulk fluid velocity. The SAM mass transport equation is as follows.

$$
\frac{\partial \rho c_{i}}{\partial t}+\nabla\left(\rho u c_{i}\right)-\nabla\left(D_{i} \nabla c_{i}\right)+\lambda_{i} \rho c_{i}=S_{i}
$$

Here, $\rho$ is the bulk liquid density, $c_{i}$ is the species concentration in units of $\mathrm{kg}$ per species per $\mathrm{kg}$ of bulk liquid, $u$ is the velocity vector, $D_{i}$ is the diffusion coefficient for the species, $\lambda_{i}$ is the decay coefficient for the species, and $S_{i}$ is the source term in units of kg per volume per second. For the new model, the drift-flux velocity is used instead of the bulk liquid velocity for the $u$ term in Eq. (15).

Two options were added to the SAM input, which are valid when transported species are defined.

- "ps_use_drift_flux" is a vector input that takes a Boolean input for each species in the model. When set to True for a species, that particular species will be transported by the drift-flux model, meaning that $u$ in Eq. (15) will be the drift-flux velocity. The default value for this input, if not provided, will be False, meaning that the bulk liquid velocity will be used for species transport.

- "ps_is_gas" is a vector input that takes a Boolean input for each species defined in the model. When set to True for a species, that particular species is defined as being a gas, which prompts SAM to calculate gas properties, including volume fraction and interfacial area. The default value for this input, if not provided, will be False, meaning that the species will be treated as a liquid.

Four new "AuxKernel" objects were added to SAM to support these new features:

"ScalarVolumeFractionAux" calculates the gas species volume fraction, "ScalarBubbleRadiusAux" calculates the representative bubble radius, "ScalarDriftVelocityAux" calculates the gas species velocity, and "ScalarInterfacialAreaAux" calculates the interfacial area of the gas species. Note that the "ScalarDriftVelocityAux" AuxKernel will be created for every species defined in the model. If the user designated the species as not using the drift-flux model, the velocity of this AuxKernel will simply be equal to the bulk velocity of the fluid. The other three AuxKernels will be created for a species only if the user indicates that that species is a gas. The name of these new variables will be based on the name of the species that has been defined. The "ScalarDriftVelocityAux" parameter will be named as species_vel, the "ScalarVolumeVractionAux" parameter will be named as species_void, the "ScalarBubbleRadiusAux" parameter will be named as species_rad, and the "ScalarInterfacialAreaAux" parameter will be named as species_aint. In all of these cases, the species base name shall be replaced by the user-provided name of the transported species. The creation of these AuxKernels will also allow Mole to obtain information from SAM that will be required for calculation of the species migration, deposition, and transport. 


\section{TEST CASES}

The primary test case was an unheated single-channel test that was developed from the hand calculation presented in Section 3. The model boundary conditions were taken from Table 1. The model was constructed using a PBOneDFluidComponent component. The friction was set to zero, and the pipe was oriented in the $y$-direction to eliminate gravitational head. In this way, the pressure is uniform throughout the component and has no impact on fluid properties. The inlet boundary condition was velocity and temperature, and the outlet boundary condition was pressure (obtained from Table 1). This model was run both with and without the drift-flux model.

It is important that the mass flow rate of the gas species be preserved regardless of whether the drift-flux or original model is used. Therefore, rather than setting an inlet species boundary condition-which would be propagated at different velocities and thus different mass flow rates depending on which model is used-a volumetric species injection rate is specified for the first node of the pipe. This appears as the $S_{i}$ term in Eq. (15). This ensures that the same amount of gas mass is injected in either case.

The species concentration comparison for both models is shown in Fig. 1. The first thing to note is that the species concentration is lower in the first node because it is being injected at that location. In nodes downstream of this, it becomes a constant value. The second thing to note is that the species concentration is different depending on which model is used. Note that the definition of species concentration becomes somewhat arbitrary for the drift-flux model case because of how the model was implemented. The mass flow rate of the species when it is transported by the liquid will be as shown in Eq. (16); however, when transported by the vapor, it is expressed as in (Eq. 17). In Eq. (16), $c_{i}$ clearly has units of $\mathrm{kg}$ of species per $\mathrm{kg}$ of liquid, but this is not the case for the gas transport scenario. Because of this, care must be taken when setting a boundary condition or interpreting the meaning of the species concentration term when using the drift-flux model. An option for circumventing this issue, and one which will be investigated further in the future, would be to use the gas density in the mass transport equation for species that are identified as a gas. In this case, $c_{i}$ would then directly represent the volume fraction.

$$
\begin{aligned}
& \dot{m}_{i}=c_{i} \rho_{l} u_{l} A, \\
& \dot{m}_{i}=c_{i} \rho_{l} u_{v} A .
\end{aligned}
$$

As a sanity check, the mass flow rate using both models was calculated and is shown in Fig. 2. Note that SAM defines the species amount as $\mathrm{kg}$ of species per $\mathrm{kg}$ of liquid, which accounts for the units in the figure. As the figure indicates, the mass flow rate for both cases is the same even though the species concentration was different. Fig. 3 shows the void distribution predicted by both models. In this case, the void fraction is quite different because of the higher gas velocity in the drift-flux model. The radius is shown in Fig. 4, which is also different; however, recall from Eq. (14) that this term depends on the relative velocity and an upper limit on bubble size was set to prevent unrealistic values. Because there is no phase slip in the base model, the bubble radius will always move to the maximum allowable value. Both the void and the bubble radius will impact the interfacial area results, which are shown in Fig. 5.

A hand calculation of the vapor velocity using the drift-flux model was performed in Section 3. In addition to this, the constitutive relations presented in Section 4 were also used to calculate the bubble radius and 


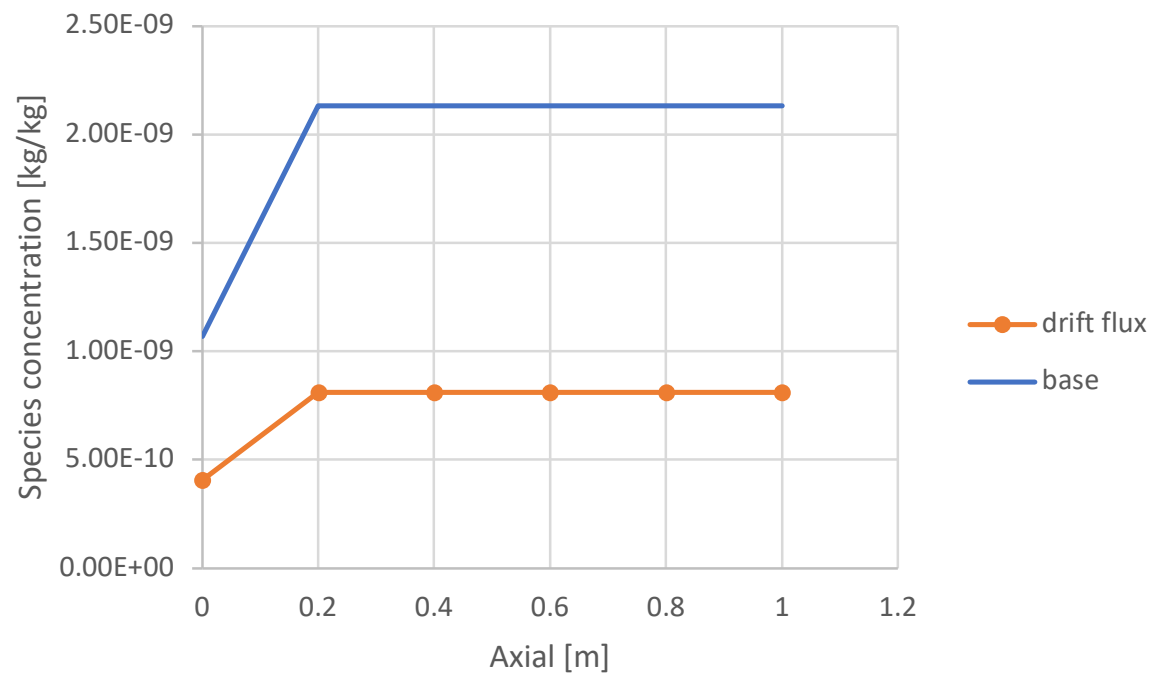

Figure 1. Axial species concentration distribution in Case 1 test using the base model and drift-flux model.

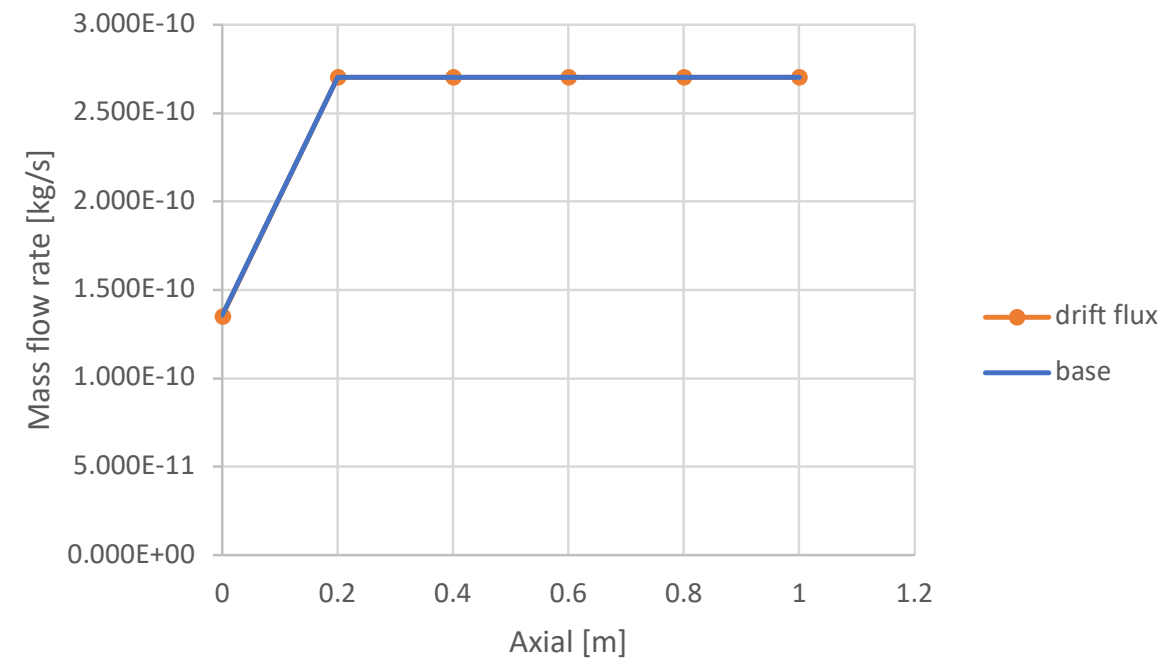

Figure 2. Axial species mass flow rate distribution in Case 1 test using the base model and drift-flux model. 


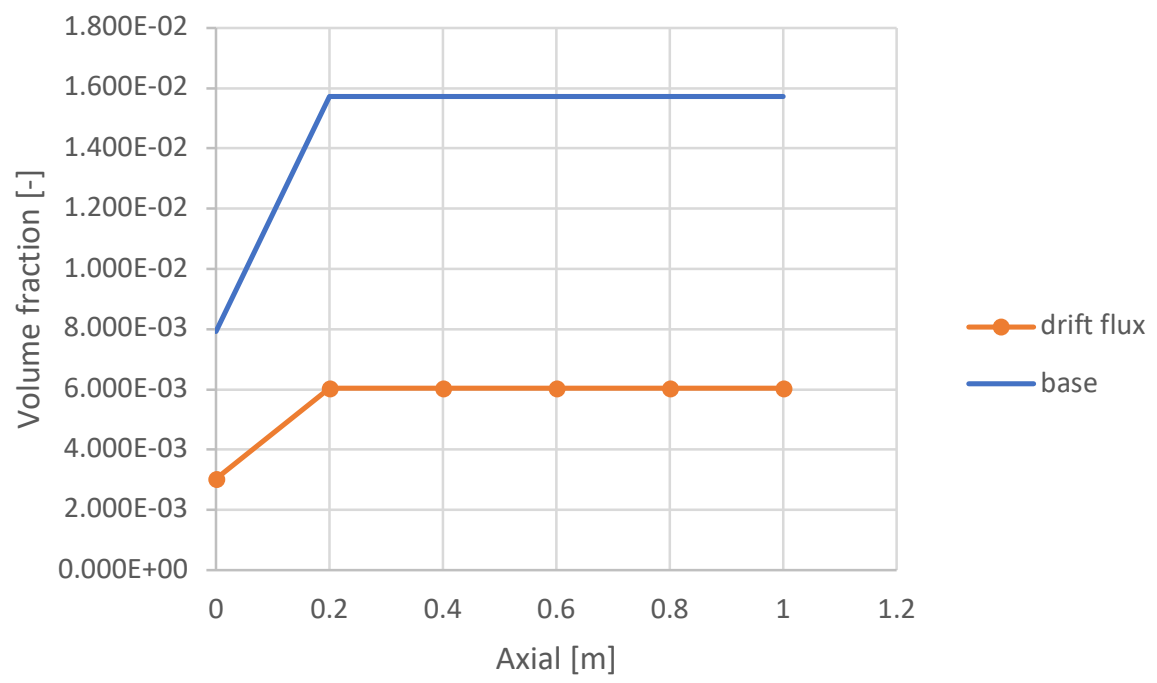

Figure 3. Axial species gas volume fraction distribution in Case 1 test using the base model and drift-flux model.

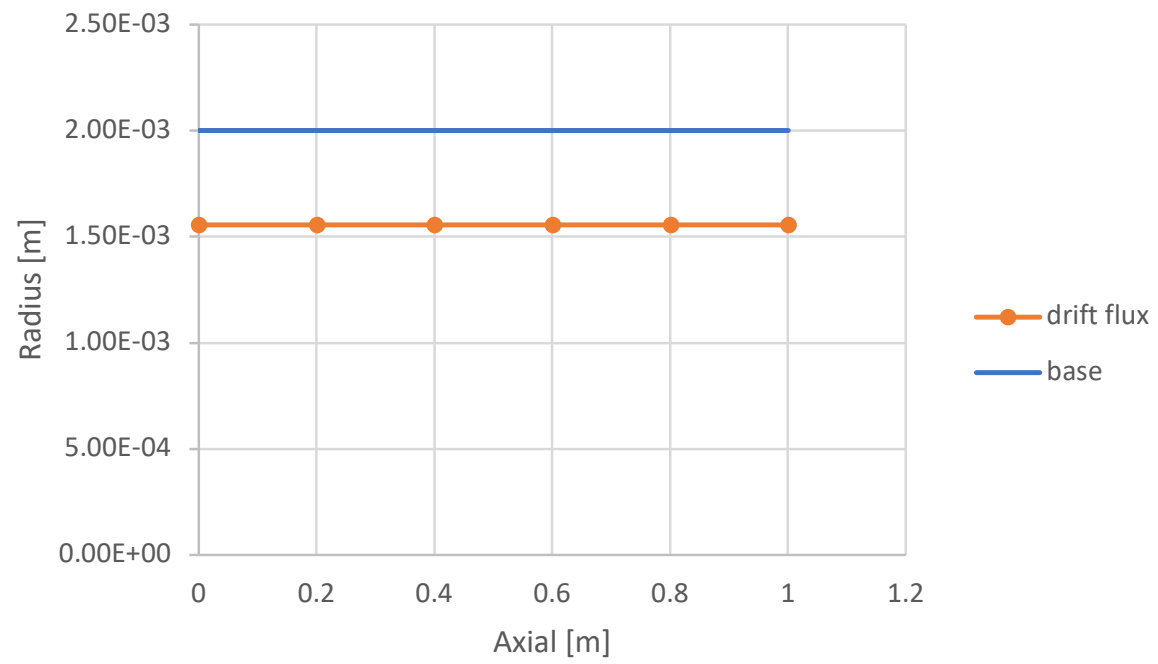

Figure 4. Axial species gas bubble radius distribution in Case 1 test using the base model and drift-flux model. 


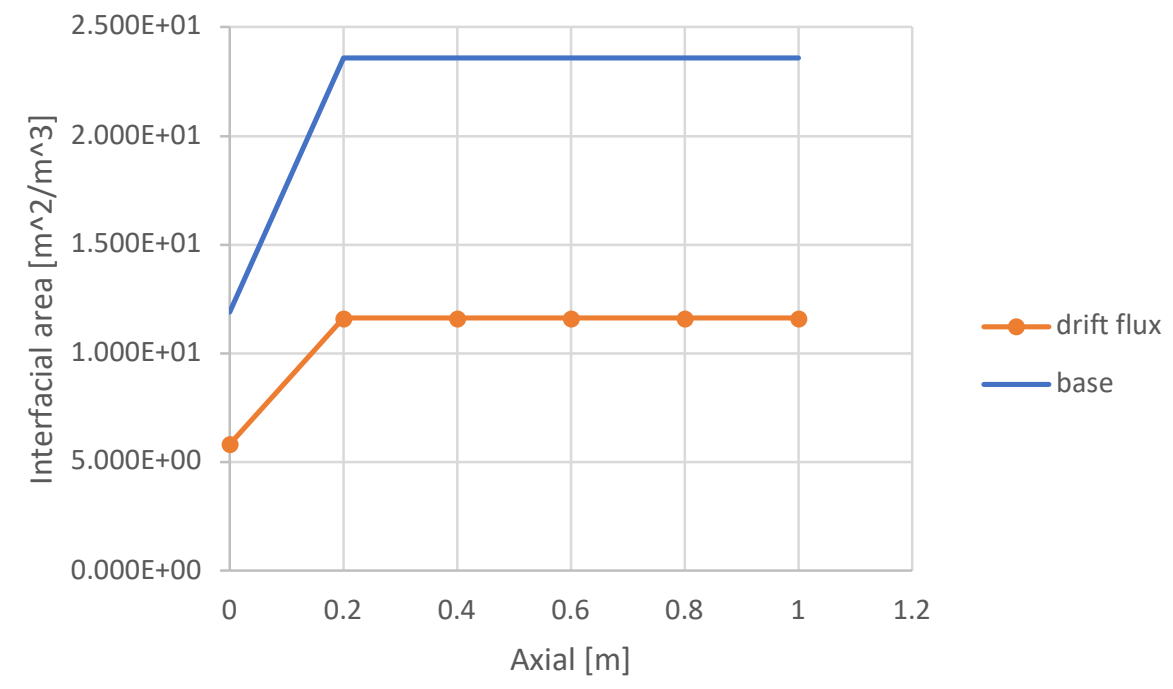

Figure 5. Axial species gas interfacial area distribution in Case 1 test using the base model and drift-flux model.

Table 2. Comparison of SAM results and hand-calculated gas phase properties for the unheated single-channel test case

\begin{tabular}{rll}
\hline Parameter & Expected & SAM \\
\hline Vapor velocity $\left(\mathrm{m} \mathrm{s}^{-1}\right)$ & 0.561 & 0.561 \\
Void & $6.04 \cdot 10^{-3}$ & $6.04 \cdot 10^{-3}$ \\
Bubble radius $(\mathrm{m})$ & $1.56 \cdot 10^{-3}$ & $1.56 \cdot 10^{-3}$ \\
Interfacial area $\left(\mathrm{m}^{2} \mathrm{~m}^{-3}\right)$ & 1.15 & 1.16 \\
\hline
\end{tabular}

interfacial area, and the drift-flux model was used to calculate the volume fraction. Table 2 shows a comparison of all hand-calculated and SAM results for this case, which are shown to be in generally good agreement.

An additional test case was created by applying a uniform heat rate of $3 \times 10^{7} \mathrm{~W} \mathrm{~m}^{-3}$ to the previous case. This led to a temperature rise of $28.1 \mathrm{~K}$, which is in line with the stated $22.2 \mathrm{~K}$ core temperature rise of the MSRE experiment. Overall, differences between the base and drift-flux models were consistent with the findings of the unheated case. A slight increase in void fraction can be observed caused by the reduction in the ratio of gas to liquid density as temperature increases over the channel, as shown in Fig. 6.

Two additional tests were added to ensure that the feature works for models that contain junctions and orientation changes. First, an unheated case was created that includes three vertical

PBOneDFluidComponents, where the first one connects to two above via a PBBranch component. An inlet boundary condition is applied to the first node of the first pipe, which supplies the same inlet flow and temperature as the first unheated case, as well as an inlet species concentration. It was verified that the outlet species concentration of the top two pipes was equal to the inlet species concentration of the first pipe when using the drift-flux model. Second, a system of two PBOneDFluidComponents-one vertical 


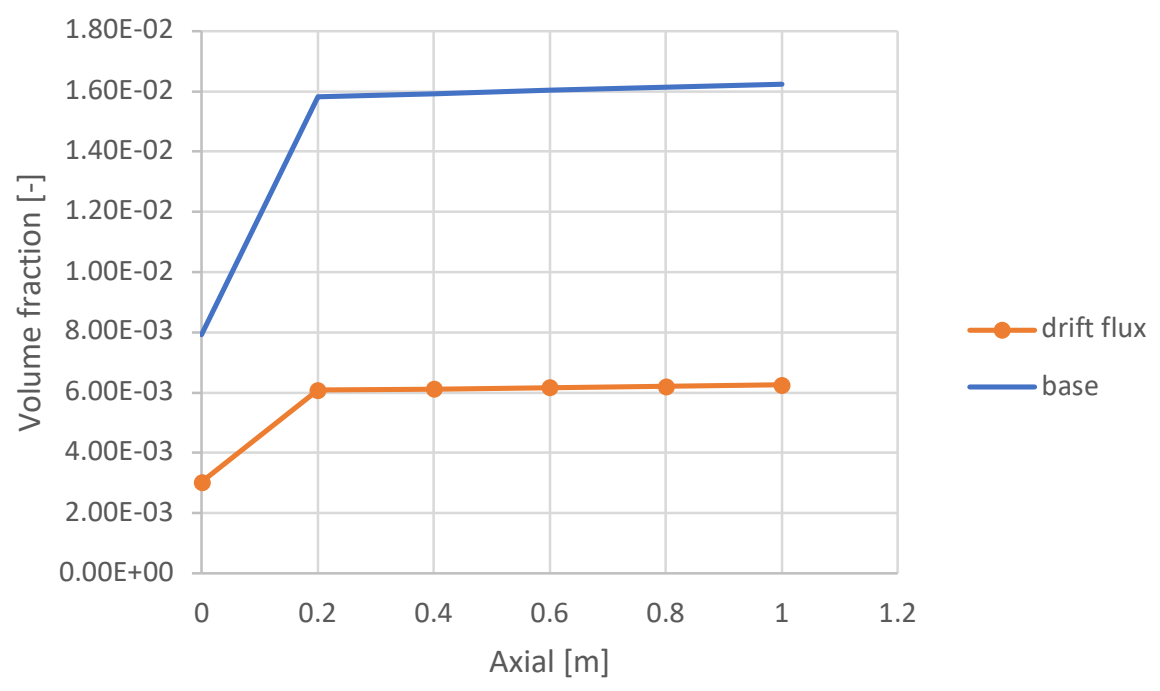

Figure 6. Axial gas volume fraction distribution in heated channel test using the base model and drift-flux model.

and one horizontal — connected by a PBSingleJunction component was created. The vertical component receives the temperature/velocity/species inlet boundary condition, and it is again verified that the outlet of the second pipe component has the same species concentration at the outlet when the drift-flux model is enabled. 


\section{CONCLUSION}

The SAM system $\mathrm{T} / \mathrm{H}$ code is being further developed to support M\&S of MSR designs. MSR designs that utilize fuel dissolved in the coolant pose unique challenges because fission products are circulated throughout the entire coolant system. Modeling this design behavior requires an M\&S tool that can accurately model the system of dissolved and gaseous fission products that appear throughout the fuel cycle. One particular fission product that is of importance is xenon-135, which will absorb neutrons and negatively impact fuel efficiency if allowed to build up to high amounts. One approach to removing xenon from the system that was found in the MSRE experiments injection of helium bubbles in the system into which the xenon will migrate. The bubbles can then be removed and run through a filtration system. Because this approach leads to a two-phase system, an investigation was performed to look into the effects of phase slip effects on gas mass transport.

A preliminary sensitivity study using the drift-flux model revealed that a potentially high slip between gas and liquid phases can be encountered because of the large density difference between the salt and the helium. Because of this, the drift-flux model was implemented into SAM for further testing. In similar ongoing work, SAM is also being coupled to the mass transport code Mole, which calculates macroscale and mesoscale diffusion, decay, and transmutation of radionuclides, as well as phase equilibrium in systems such as the MSR. In this coupling, it is intended that SAM will provide the velocity distribution and interfacial area for transported species in the MSR system. The drift-flux model that has been implemented into SAM will be tested in Mole to determine its impact in a multiphysics sense.

Testing of the drift-flux model has indicated that it agrees well with hand calculations for simple geometries and that it behaves correctly for slightly more complex models, including multiple flow components connected by junctions. In future work, testing will be performed for more complex geometry systems that are representative of the MSRE flow loop, and support will be provided for the coupling to Mole. Furthermore, assumptions that were made during this preliminary work-including assuming that the gas is always helium and that the surface tension is fixed to a constant value found for FLiBe-will be removed by implementing more accurate fluid properties and by providing the user the flexibility to use them via the SAM input file. 


\section{REFERENCES}

D. E. Chavez. Flow visualization of two-phase flow in molten-salt: Helium bubbling in lithium fluoride-sodium fluoride-potassium fluoride (lif-na-kf). Master's thesis, Texas A\&M University, 2020. URL http://hdl.handle.net/1969.1/192215.

J. Engel and R. Steffy. Xenon behavior in the molten salt reactor experiment. Technical Report ORNL-TM-3464, Oak Ridge National Laboratory, 1971.

R. Hu, L. Zou, G. Hu, D. Nunez, T. Mui, and T. Fei. Sam theory manual. Technical Report ANL/NSE-17/4 Rev. 1, Argonne National Laboratory, 2021.

M. Ishii. One-dimensional drift-flux model and constitutive equations for relative motion between phases in various two-phase flow regimes. Technical Report ANL-77-47, Argonne National Laboratory, 1977.

R. J. Kedl. The migration of a class of fission products (noble metals) in the molten-salt reactor experiment. Technical Report ORNL-TM-3884, Oak Ridge National Laboratory, 1972.

K. O. Lee, Z. Taylor, and B. Collins. Implementation of decay and liquid-gas mass transfer to enhance mass accountancy capabilities in mole. Technical Report ORNL/TM-2021/2161, Oak Ridge National Laboratory, 2021.

R. Salko, M. Avramova, A. Wysocki, A. Toptan, J. Hu, N. Porter, T. Blyth, C. Dances, A. Gomez, C. Jernigan, J. Kelly, and A. Abarca. CTF Theory Manual. Consortium for Advanced Simulation of Light Water Reactors, 2019.

M. Sohal, M. Ebner, P. Sabharwall, and P. Sharpe. Engineering database of liquid salt thermophysical and thermochemical properties. Technical Report INL/EXT-10-18297, Idaho National Laboratory, 2010.

Zack Taylor, Robert Salko, Aaron M. Graham, Benjamin S. Collins, and G. Ivan Maldonado. Implementation of two-phase gas transport into vera for molten salt reactor analysis. Annals of Nuclear Energy, 165:108672, 2022. ISSN 0306-4549. doi: https://doi.org/10.1016/j.anucene.2021.108672. URL https://www.sciencedirect.com/science/article/pii/S030645492100548X.

N. Zuber and J.A. Findlay. Average volumetric concentration in two-phase flow systems. Journal of Heat Transfer, 87, 1965. 